\title{
The Millennial Mum - Technology Use by New Mothers
}

\author{
Dr Suzanne Prior \\ Abertay University \\ Bell Street, Dundee, UK \\ s.prior@abertay.ac.uk
}

\begin{abstract}
Becoming a mother presents a woman with new challenges and a need to access new sources of information. This work considers the increase in the use of online parenting support as the first group of millennials become parents. Initial results from a survey comparing the use of technology pre and post the experience of childbirth is presented.
\end{abstract}

The survey reveals that mothers are likely to increase the time they spend online and are strongly motivated by seeking social contact. The paper concludes by considering the implications of this survey for healthcare professionals wishing to give information and support through online media and suggests how $\mathrm{HCl}$ professionals can become involved in this work.

Motherhood, Millennials, Social Networks, eHealth

\section{INTRODUCTION \& BACKGROUND}

The process of pregnancy and the early months of being a parent are fraught with challenges and often require input from a variety of health professionals. Unsurprisingly there have been many different interventions designed by researchers to support women who face challenge during this period of transition. In particular this has come from psychology and medical fields with potential treatments including medical drugs, cognitive behavioural therapy and structured social support amongst others.

The $\mathrm{HCl}$ field has in the past been slower to take notice of this period of transition, the majority of the focus on family/parental involvement in technology has focussed on the use of technology by children and teenagers and how their parents can respond to this. Now we have reached a point in time in which the millennial generation - generally accepted to be those people born between 1980 and 2000 (Strauss, Howe 1991) - and original digital natives, who were the teenagers in these original studies are entering their late 20 s and 30 s and becoming parents themselves. There is now a need to consider how technology is impacting on this experience from a $\mathrm{HCl}$ perspective to complement and collaborate with research from other fields in this area.

This requires us as $\mathrm{HCl}$ researchers to begin to shift our focus from examining only the experiences of parents who have not grown up with technology, and instead also explore the experiences of those who have grown up with it, who used technology as part of their everyday lives prior to becoming parents and who continue to use it during the early years of childrearing. Decisions made by mothers during this time have long term implications upon the health of their child, and health providers have begun to develop interventions in this area. However, an understanding of how new mothers use and are motivated to use technology is still limited and as yet an underexplored area.

Recent years have started to show an understanding of this development and we have seen investigation in this area. The $2013 \mathrm{CHI}$ Workshop on 'Motherhood and HCl' (Balaam et al. 2013) is representative of this new area of interest. Gibson and Hanson (2013) conducted an ethnographic study into new mother's use of technology. Centred around five mother and baby social groups they found that new mothers used their smart phones to go online for emotional wellbeing more than they were used for traditional forms of communication. Work done by De Choudbury (2013) has examined how social media posts by new mothers on Twitter can be analysed in order to predict their risk of postnatal depression.

This early stage study proposes to expand upon these previous studies and explore how these digital natives use a variety of online technology during the transition to motherhood. 


\section{METHODOLOGY}

A survey was developed to be completed online. This survey was available for one month between November and December 2015. This study was approved by the institutional ethics committee and the online survey began with an informed consent process. Inclusion criteria for the survey were being a mother of a child under the age of 2 years old and having used online technology during the first 6 months of their child's life.

The survey was composed of eleven questions. Five questions focussed on demographic information, two on their experiences of using technology prenatally, and four on their experience of technology postnatally. During the time period that the survey was available for 103 participants took part. The average age of a participant's youngest child within the survey was 15 months (minimum 3 months, maximum 24 months). The maximum number of children any participant had was four with an average of one. The majority of participants were between the ages of 26 and 30 ( $n=68)$ meaning that they fitted into the millennial generation, this is slightly lower than the national average age of 30 for first time mothers.

\section{DISCUSSION}

One key finding from the initial analysis of the quantitative questions was that new mothers increased the time they spent online by 19 minutes per day $(d f=99, p<0.05)$. A thematic analysis of the results of the survey revealed 7 themes (Maintenance of contact with the world outside of the "Baby Bubble"; To pass time while breastfeeding the baby; To develop new relationships and support other new mothers; Searching Internet Websites for Information and Reassurance; Updating contacts on information relating to baby and taking/sharing photos; To pass time separately from tasks related to child rearing, and; To carry out daily tasks such as shopping more conveniently.). Several themes pointed to a desire for socialisation. This suggests that despite having more demands upon their time, mothers are looking to the internet to provide the social support they require rather than more traditional sources.

The free text motivations made frequent references to different forms of socialisation and a desire to seek this out. There was a decrease in the number of participants using Twitter following childbirth; this is interesting given the ongoing debate as to whether Twitter is a social network or a form of news broadcasting. Twitter provides less opportunity for group discussions due to the character restriction and is more public than other social networks. In contrast many parenting groups on Facebook are either set to private (meaning that only members can see what is being posted) or secret (meaning that another member has to invite a user to become a member - the group will not appear in a Facebook search). This suggests that it is personal connections and the wish to strengthen these which is motivating mothers to go online.

Mothers are using these social networks to get reassurance on topics which they would previously have looked for from members of their physical community. The reduction in support from local health professionals such as General Practitioners and health visitors (Gordon-Walker 2014) has been occurring concurrently to the growth in online parenting support and may be a factor in this increase. This social support has the potential to reassure mothers but those wishing to develop digital tools should give careful consideration as to how health guidance is given. Survey participants made reference to asking for advice on how to treat a child's symptoms or for suggestions as to the cause of a rash. Incorrect medical advice in this area could have potentially fatal consequences. Of course this has been challenge in other eHealth studies, but is of particular concern in this area.

The development of new relationships suggests the formation of community is important. As discussed previously this can be seen in contrast to the breakdown in communities in the physical world (Swigger 2012). This is important for medical professionals, looking to provide new mothers with social support, to consider.

\section{CONCLUSION}

Recent years have seen a growth in online resources for new parents and this is only likely to increase as the millennials continue to age and become parents. This survey has demonstrated that during the period of time following the birth of a child, mothers increase the time they spend online. They are motivated to do this for a variety of reasons but in particular for socialisation. This suggests that there is potential to harness these new forms of community when delivering healthcare interventions and we strongly encourage $\mathrm{HCl}$ researchers to consider entering discussions around the challenges and opportunities in this area.

Health providers have already begun to take advantage of this familiarity with technology and provided detailed medical advice through their websites. However as this study shows, many mothers are motivated by making connections and socialising with others in a similar situation. More work is now needed to fully understand the reasons behind this desire for socialisation and what the impact of this is upon those looking to design eHealth interventions for this user group. 


\section{REFERENCES}

Balaam, M., Robertson, J., Fitzpatrick, G., Say, R., Hayes, G., Mazmanian, M. \& Parmar, B. (2013), Motherhood and $\mathrm{HCl}$. In $\mathrm{CHI}$ '13 Extended Abstracts on Human Factors in Computing Systems. Paris, France (27 April - 2 May 2013) pp. 3215.

De Choudhury, M., Counts, S. \& Horvitz, E. (2013), Predicting postpartum changes in emotion and behaviour via social media. In Proceedings of the SIGCHI Conference on Human Factors in Computing Systems - CHI '13. Paris, France (27 April - 2 May 2013), pp. 3267.

Gibson, L. \& Hanson, V.L. (2013), Digital Motherhood. In Proceedings of the SIGCHI Conference on Human Factors in Computing Systems - CHI '13. Paris, France (27 April - 2 May 2013), pp. 313.
Gordon-Walker, M. (2014), Available from http://www.theguardian.com/commentisfree/201 4/jul/08/health-system-failing-new-motherspostnatal-depression-nhs (6 June 2016)

Strauss, W.A. \& Howe, N. (1991), Generations: the history of America's future, 1584-2069 Morrow, New York, USA.

Swigger, N. (2013), "The Online Citizen: Is Social Media Changing Citizens' Beliefs About Democratic Values?", Political Behavior, vol. 35, no. 3, pp. 589 - 603. 